\title{
Measuring Sandy Bottom Dynamics by Exploiting Depth from Stereo Video Sequences
}

\author{
Rosaria E. Musumeci ${ }^{1}$, Giovanni M. Farinella ${ }^{2}$, Enrico Foti ${ }^{1}$, \\ Sebastiano Battiato ${ }^{2}$, Thor U. Petersen ${ }^{3}$, and B. Mutlu Sumer ${ }^{3}$ \\ 1 Dept. of Civil and Environmental Engineering, University of Catania, IT \\ 2 Dept. of Mathematics and Computer Science, University of Catania, IT \\ 3 DTU Mekanik, Section for Fluid Mechanics, Coastal and Maritime Engineering, \\ Technical University of Denmark, DK
}

\begin{abstract}
In this paper an imaging system for measuring sandy bottom dynamics is proposed. The system exploits stereo sequences and projected laser beams to build the 3D shape of the sandy bottom during time. The reconstruction is used by experts of the field to perform accurate measurements and analysis in the study of the final equilibrium conditions of sea bottoms in the presence of water flows. Results obtained by processing data acquired in hydraulic laboratory confirm the effectiveness of the system which makes simple and fast the understanding of the sandy bottom dynamics and the related equilibrium phenomena.
\end{abstract}

Keywords: Sandy bottom dynamics, Stereo system.

\section{Introduction}

The investigation of the three-dimensional morphological evolution of coastal sandy bottoms under the action of waves and currents is particularly relevant in the fields of physical oceanography and coastal engineering. For example, a detailed knowledge of scour processes at the foundation of coastal structures is fundamental to predict damages and failures potential of the structures themselves. Such a kind of studies are usually tackled by means of laboratory investigations, where all the complexity of the involved phenomena can be well controlled and reduced to simpler and reproducible conditions [1]. A typical experimental setup is shown in Figure 1 .

In hydraulic wave flumes, mechanical instruments are often used to determine bottom evolution [2], however optical instruments are becoming more and more popular due to their noninvasiveness with respect to the experiments, i.e., the fact that it is possible to observe the dynamics of the bottom induced by the flow without generating spurious disturbance to the flow itself. This is one of the aims, for instance, of the approach proposed in [3] where the authors used a single mini waterproof camera coupled with a $45^{\circ}$ mirror located within a transparent pile to monitor at a single point the maximum scour depth at the basis of a vertical cylinder. 


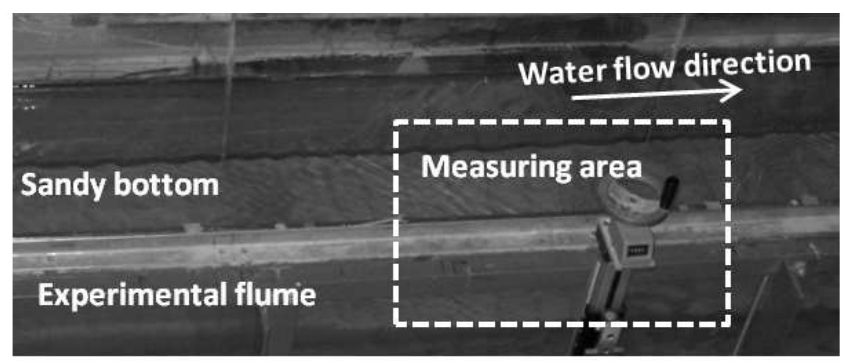

Fig. 1. View of the experimental setup

In order to gain knowledge of the three-dimensional characteristics of the coastal bottom morphology, several 3D based approaches have been recently proposed, ranging from commercial 3D laser scanners to more sophisticated stereo pairs techniques [4-7]. Unfortunately such optical methods are usually limited to measure the phenomena under consideration in dry condition, i.e., either the wave flume need to be stopped and drained either the measuring area is located in the dry part of the shore. Moreover the images of sandy bottom are structureless and it is difficult to find corresponding points in stereo pair images to be used to reconstruct the sandy bottom and to track interesting points and areas to understand their evolution in time. A recent study [4] have proposed a stereoscopic technique able to measure in the dry part of the swash zone (i.e., in the part of the beach which is alternately wet and dry) rapid variations of the sandy bed elevation based on the use of Particle Tracking Velocimetry (PTV) algorithms. A grid of light dots (each dot was $2 \mathrm{~cm}$ wide) projected on the dry part of the swash zone has been employed to add some recognizable structure to be tracked in the images.

Obtaining 3D measurements of the bed morphology undewater is much more difficult, due to problems both in image formation and image aquisition (see Figure 1). Indeed, due to the small size of the sand grains, $O\left(10^{1} \mu \mathrm{m}\right)$, and to the limited variability of colours, the images of sandy bottoms are "structure free", hence making difficult the matching of the points of the different point of view (i.e., correspondence problem in stereo systems). On the other hand it is preferable that the cameras are located below the water level and relatively far from the observing area, to avoid variable refraction and reflection disturbances from the moving water surfaces and undesired localized effects due to the presence of the optical equipment. Notice that classic computer vision techniques adopting interest feature points (like SIFT [8]) are not suitable to solve the matching problem in this context since the unstructured material observed into the scene (i.e., the sand) due to the high variability of the background induced by the water waves and the related reflection.

To deal with such problems, a stereoscopic technique with the cameras located in front of the glass wall of the flume below the still water level has been proposed in [5]. A grid of laser dots projected on the sandy bottom has been used to allow manual correspondences (made by the experts) between points 
observed by the stereo pairs and hence to perform 3D measurements. The main advantage of the technique is the possibility to perform 3D dynamic measurements of the bottom evolution (i.e., 3D depth is generated from stereo video) without disturbing the water flow. Such a technique was extended in [6] considering large scale applications in the swash zone region. In that case, not only a much greater number of dots was deemed necessary, but also longer time series were recorded up to equilibrium conditions. The technique has been also recently applied in [7] to investigate the backfilling process of a scour at the base of vertical cylinder. In this last work the possibility to measure the $3 \mathrm{D}$ dynamics of the bottom evolution has been fundamental to demonstrate that wave-induced scours and wave-induced backfillings of a current-scour lead to the same equilibrium conditions. However, although quite accurate from the measuring point of view, the procedure used in [5-7] to build the 3D maps is not easily applicable to the analysis of a large number of images (e.g. frames of long video pairs), required for an accurate analysis of the time evolution of the process. Indeed, both the automatic detection of hundreds of grid laser dots contained in each image and the matching of the stereo pairs are carried out manually by the experts. This process becomes impossible to do by considering hours of acquisition at $4 \mathrm{fps}$, as the experimental setting of this paper. Hence, in order to become an useful and versatile measurement instrument for sandy bottom evolution the aforementioned technique needs to be made automatic.

Building on previous works [5-7], in this paper an automatic system for measuring sandy bottom dynamics from stereo image sequences is proposed. After system calibration, the projected laser beams are detected on the stereo pair frames through an image processing pipeline. Exploiting the calibration parameters, the detected dots on the left image are matched with the ones on the right by projecting their coordinates and considering weak perspective assumption. Then the 3D maps are hence obtained by triangulation and the analysis is performed.

The paper is organized as follows: in Section 2 the proposed system is described by focusing on (i) the automatic detection of the laser dots onto the gathered images of the sandy bottom, and (ii) the automatic stereo matching of the dots on the stereo pairs. Section 3 discusses the experimental setting and summarize the results. Finally, conclusions and hints for future works are given in Section 4.

\section{Proposed System}

As it is standard in stereo vision approaches, the proposed technique can be schematized in the following three stages: (i) stereo image acquisition; (ii) matching of the corresponding points; (iii) 3D measurement and analysis.

The main objective of the instrumental setting used in this work is to obtain "suitable" images to make correspondences of points of the sandy bottom morphology throughout the experiment. Moreover, to make accurate measurements of the bottom dynamics the depth of specific points (e.g., evolution of points around a vertical pile) should be reconstructed in time. This is achieved by projecting a grid of laser points onto the sandy bottom. Figure 1 shows a typical 


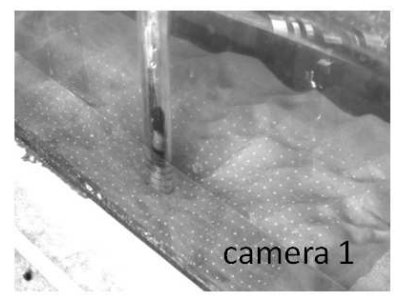

(a)

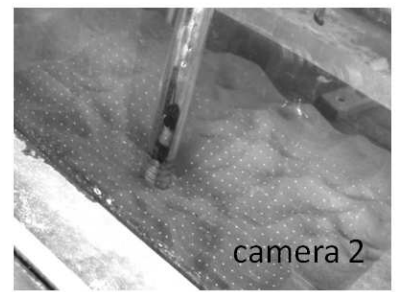

(b)

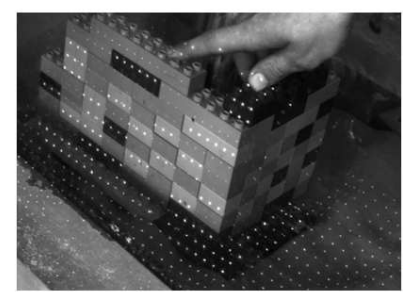

(c)

Fig. 2. (a) and (b): stereo pairs related to the experimental settings. The projected laser dots onto the sandy bottom and the the scour around a vertical pile are visible. (c) $3 \mathrm{D}$ rig used during calibration.

view of the experimental setup, while Figure 2( $a$ ) and $b$ shows an example of a stereo images where the laser grid points are visible. The light dots become an optical well-defined point measuring stations on the sandy bottom, and also allows to add points to be matched between the stereo images.

In a previous work [5], Gaussian lenses have been used during acquisition. Here we follow the protocol of [6] where non-Gaussian lenses have been exploited to obtain an uniform density distribution of light with sharp ends. External lighting conditions of the hydraulic laboratory are also very important. In the ideal condition a dark room in which only the laser beams can be seen simplify the detection of the points. Unfortunately those conditions are difficult to be obtained in hydraulic laboratories, where for safety reason windows cannot usually be shaded to get complete darkness. Spurious reflections, due to the flume's glass walls or to the moving free surface of water, can also affect the quality of the images. It follows that the images must be carefully analyzed in order to reject points which do not belong to the laser grid projected onto the bed. As can be assessed by visual inspection of Figure 2 $(a)$ and (b), there are many source of variability in the images which make difficult both, dots detection (e.g., there are many false positive dots coming from reflection) and matching.

Being a stereoscopic approach, the image acquisition is obviously performed by using two cameras which simultaneously record videos of the sandy bottom evolution. In order to avoid any disturbance to the flow, the cameras are located outside of the flume, in front of glass walls in the correspondence of the measuring area. Due to the fact that in hydraulic laboratories several optical obstacles are usually present (e.g., steel frames, structural elements, other measuring instruments, etc.), the technique has been developed in such a way that there are no constraints on the relative position of the two cameras. The only requirement is that both cameras should have a "vision" as much complete as possible of the phenomenon under investigation.

About the camera calibration, while [5] and [6] used a direct linear transformation (DLT) $\sqrt{9}, 10]$, in the present work a four-step camera calibration procedure is performed [11]. Traditionally, several views of a 2D planar rig are adopted during calibration [12]. To facilitate the operations within the flume filled with water, the 


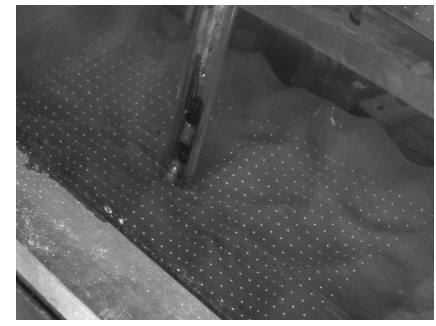

(a)

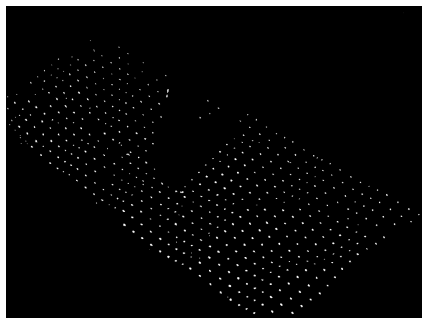

(c)

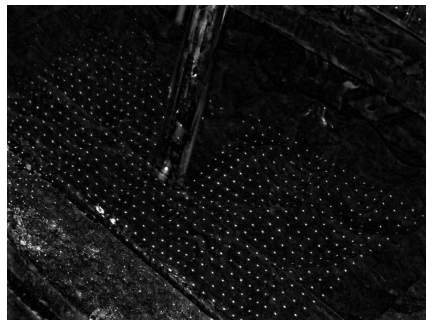

(b)

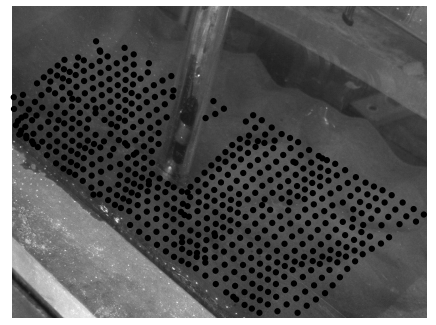

(d)

Fig. 3. Detection of laser dots. (a) Original image; (b) Filtered image; (c) Top-hat transform; d) Dots vs. other separation; e) Detected laser dots.

stereo images of a 3D calibration rig are used (see Figure $2(c)$ ). The rig has known dimensions, which can be modified according to the dimensions and shape of the investigated phenomenon to properly calibrate the stereo system in order to obtain the required accuracy in the measurements. Once, the calibration of the stereo system is done, the laser dots should be detected and matched in the image pairs to obtain the correspondences useful to build the 3D map of the sandy bottom at varying of time. The following subsections illustrate the techniques implemented for making automatic the laser dot detection and matching.

\subsection{Detection of Laser Dots}

The automatic detection of the light dots position on the images is obtained by means of an image processing pipeline involving a sequence of filtering, morphological operations and foreground (i.e., dots) versus background separation procedures. An example of the images obtained at the various steps of the pipeline is shown in Figure 3. Note that, due to the high variability and artifacts introduced by the reflection, noisy input, etc., this task is not so trivial as expected.

As first, each image is filtered by means of a Gaussian kernel to remove noise (see Figure 3 $(b)$ ). Then, in order to correct uneven illumination and to enhance the intensity of the laser dots, a morphological top-hat transform [13] is carried out by means of a disk structuring element (see Figure $3(c)$ ). The results of such an operation is hence binarized through a thresholding procedure to separate dots (i.e., foreground versus background). Therefore a flood-fill operation 14] of the obtained binary image is applied to fill holes, (i.e., to fill the background pixels that cannot be reached by filling in from the edge of the images). Finally, the 


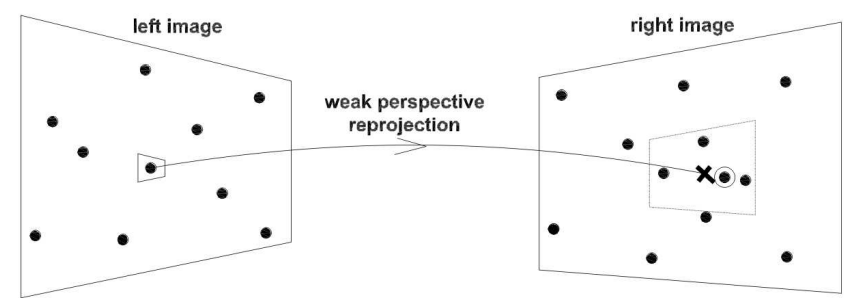

Fig. 4. Closest neighbour matching considering weak perspective

segmented spots (i.e., the connected elements on the image) are analyzed with respect to their shapes (through geometric constraints, such as area, etc.) in order to retain only the laser dots (see Figure $3(d)$ ). Then, the centroid coordinates of all the detected dots are determined and stored.

The parameters used in the aforementioned pipeline have been learned on the pair of images used for the stereo calibration procedure. Specifically, for those images a manual labeling has been performed and the parameters have been learned with a search procedure on a quantized version of the parameter space.

\subsection{Stereo Matching}

In a previous work [6], it was shown how standard methods used for stereo matching (e.g., correlation) fail to properly recover the sandy bottom geometry, due to the lack of structures useful to deal with the correspondence problem. As we have experimented in this context, the classic approach to match invariant feature points (e.g., SIFT [8]) in the pair images has problems due to the high variability and presence of artifacts generated by the reflection and to the periodic patterns and deformation present in the sandy texture and in the waves induced by the experiments.

At this stage we have the detected dots from the pipeline described in previous section. Also, since the cameras have been stereo calibrated, all the intrinsic and extrinsic parameters of the stereo system are known. Therefore, in the present work, the problem of stereo matching is solved by means of a Closest Neighbour Method (CNM), i.e., through a purely geometric approach, based on the distance of the reprojected points and making the assumption of weak-perspective model [15]. A sketch of the proposed procedure is shown in Figure 4. In our case, the approximation to a weak perspective model is possible and profitable since the dimension of the measuring area is relatively small compared to the distance of the cameras from it (i.e., the average variation of the depth of the measured area is very small with respect to the average distances of points). Hence, a constant size window is introduced to select a set of dots on the right image which are neighbours of the reprojection. Within such a set, the dot which is closest to the reprojection is chosen as the matching point. Subsequently, a series of checks are performed to guarantee a biunique correspondence of the matchings and hence remove false positive and wrong matchings. 


\subsection{D Sandy Bottom Reconstruction and Analyses}

Once the matching pairs are obtained the depth of the sandy bottom is reconstructed by using triangulation [15]. Moreover, in order to obtain a smooth 3D depth maps over time, a linear interpolation of the 3D dots coordinates related the sandy bed is carried out. The morphology of the sandy bottom surface is enlightened by means of contour lines. Since the depth map is built for each stereo pairs of the video streams, the 3D dynamics of the sandy bottom can be analyzed following the dots of interest for the specific experiment (e.g, points around the pile).

\section{$3 \quad$ Experimental Setting and Results}

In the present work, the proposed system has been applied to a laboratory analysis of the evolution of the scour which occurs at the foundation of an offshore structure due to marine currents and the refilling (or backfilling) of such a scour hole generated by the subsequent action of wind waves. From the engineering point of view, a throughout three-dimensional understanding of the phenomenon is particularly important for protecting the foundations of off-shore structures (e.g., wind turbines, oil platforms, etc.) from erosion.

The experiments have been carried out at the hydraulic laboratory of the Section for Fluid Mechanics, Coastal and Maritime Engineering of the DTU Mekanik of the Technical University of Denmark in a wave-current flume, which is $28 m$ long, $0.6 m$ wide and $0.8 m$ deep. Fine sand was used during the experiments, having a median diameter $d_{50}=0.17 \mathrm{~mm}$ and gradation equal to 1.3 . The water depth was $h=0.4 \mathrm{~m}$. The diameter of the plexiglass vertical circular pile used to investigate scour and backfilling was $D=40 \mathrm{~mm}$. In Figure 3( $a$ ) is possible to observe the aforementioned setting as seen from the right camera.

The evolution of the bottom morphology was recorded starting from flat bottom conditions up to current- or wave-induced equilibrium scour and backfilling by means of two UI-2250RE-M monochrome cameras, located outside of the wave flume (see Figure 2(a) and $(b)$ ). The grid of light points was obtained by means of two $20 \mathrm{~mW}$ Lasiris SNF 660-nm laser generators (in order to cover the measuring area) coupled with two GMN31614MNC-1 Goyo Optical Inc. diffractive lenses with $1.6 \mathrm{~mm}$ focal length. Each laser produced a square grid of $19 \times 19$ equally spaced laser beams, with a total of 722 dots projected onto the sandy bottom. Some of the laser dots were not visible due to the pile shadow.

A total of 137 video pairs were recorded at $4 \mathrm{fps}$, using an image size of $1600 \times 1200$ pixels. The duration of each video was in the range of 2 to 15 minutes. The longest video pairs were recorded at the beginning of the experiment to monitor the initial stages of the evolution which are the fastest, then video pairs were taken each 10 minutes up to an equilibrium condition was reached, after $2 \div 4$ hours. One of the main motivation of the present work is that, the manual treatment of the above data is extremely time consuming, since the analysis of one image of a single stereo pair may take up to 2 hour. Moreover, due to the 
small size of the light dots, the manual detection and matching are prone to material errors of the operator.

Here we report one case study related the investigation of the dynamics of sediment transport around a vertical cylinder [7] (see Figure 5). The considered images have been chosen in such a way to be representative of several stages of the bottom evolution.
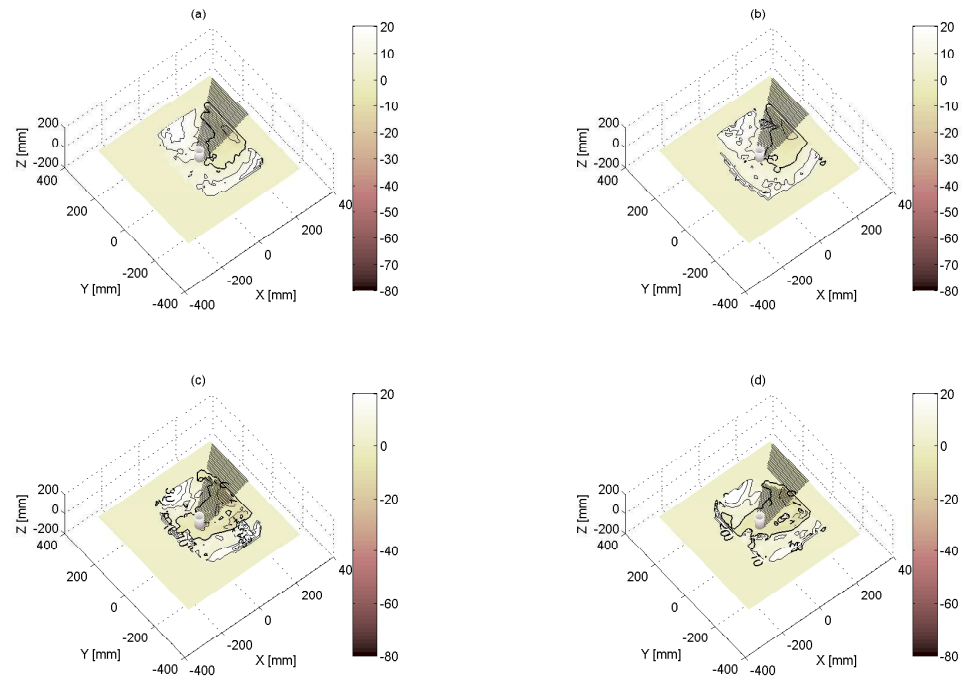

Fig. 5. 3D depth maps of the sandy bed: (a) flat bottom condition (Stereo Pair 1) as recovered manually [7]; (c) flat bottom condition (Stereo Pair 1) as recovered by the proposed automatic pipeline; (b) backfilling at equilibrium (Stereo Pair 5) as recovered manually [7]; (d) backfilling at equilibrium (Stereo Pair 5) as recovered by the proposed automatic pipeline

By defining $T$ as the time scale of the backfilling process, the set of pairs related the case study refer to the following experimental conditions:

- Stereo Pair 1: initially flat bottom;

- Stereo Pair 2: current-induced scour at equilibrium;

- Stereo Pair 3: wave-induced backfilling after a time equal to $T / 2$;

- Stereo Pair 4: wave-induced backfilling after a time equal to $T$;

- Stereo Pair 5: wave-induced backfilling at equilibrium;

To perform an objective evaluation of the proposed system, the dots in the images related the case study under evaluation were manually labeled and matched. The total number of manually labeled dots is 5610 . The final number of manually labeled matchings is 2660 . The overall detection rate obtained with the pipeline summarised in Section 2.1 was $0.83 \%$ with an average rate per image of $0.825 \%$. The matching rate obtained considering the corrected detected points was $0.734 \%$ for the equilibrium condition state. The most important fact is that the interesting dots useful for the investigation of the dynamics of sediment transport around the pile were well detected and matched allowing the study 
of the flume at the equilibrium backfilling condition (Condition related Stereo Pair 5 in the list above). The results obtained can be considered satisfactory to perform the analysis, although further improvement is deemed necessary to reach better performances of the matching in the case of sandy bottom morphologies characterized by high steepness and high reflection noise and artifacts (e.g., condition related to the Stereo Pair 2 in the above list).

Once the matching is done, the 3D map of the sandy bottom is reconstructed. Examples of the 3D maps obtained from both manually labeled process [7] and from proposed method are reported in Figure 5 for Stereo Pair 1 and Stereo Pair 5 , which corresponds respectively to the flat bed conditions and the wave-induced backfilling at equilibrium.

In Figure 6, the cross-sections of the sandy bottom along the central axis of the wave flume obtained by the manual reconstruction [7] and the one performed with the proposed automatic procedure are compared. The data are referred to the equilibrium condition of wave-induced backfilling. The independent measurement of the position of the sandy bottom (i.e., the ground-truth) as been recovered by a waterproof minicamera coupled with a $45^{\circ}$ mirror [7] is also reported in the plots (i.e., the red dot in Figure 6). This result shows how the proposed method is able to accurately reconstruct the 3D sandy surface and hence can be used to measure the interesting quantities of morphological analyses, such as the measurement of the maximum scour depth. The results obtained with the proposed method outperforms the previous results obtained through manual approach [7].
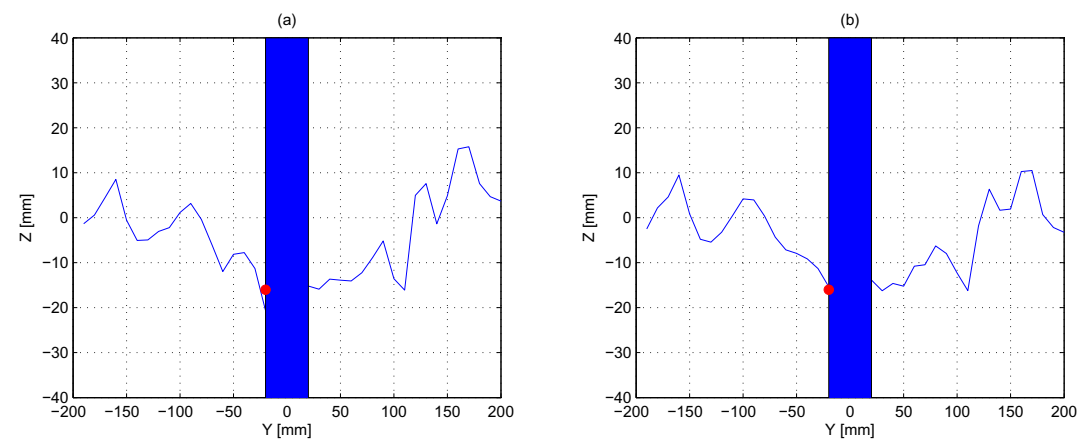

Fig. 6. Cross-section along the central axis of the flume at the equilibrium backfilling condition: (a) result recovered by using manual reconstruction [7]; (b) result obtained by the proposed automatic pipeline. The red dot indicates the ground-truth related the bed position [7]. The proposed method obtains the best result.

\section{Conclusions and Future Works}

In this paper a system for measuring quantities useful for sandy bottom morphological analyses over time has been presented. The system makes use of stereo vision and projected laser beams which are automatically detected and matched 
to find the correspondences useful for the $3 \mathrm{D}$ sandy surface reconstruction. The results obtained in measuring the final equilibrium conditions by processing data acquired in hydraulic laboratory confirm the effectiveness of the system which makes effortless for the expert the understanding of the sandy bottom dynamics phenomena. An improvement of the proposed method is under development to cope with the limits due to the weak perspective model assumption used here for reprojection. Moreover, future works will be devoted to test the performances of recent hardware (e.g., Kinect [16]) for the 3D sandy bottom reconstruction.

Acknowledgments. The research described in this manuscript was supported by the EC project HYDRALAB IV (contract no. 261520) and by the Italian Ministry for Education, University and Research funded PRIN 2010-2011 HYDROCAR (Prot. no. 20104J2Y8M_003).

\section{References}

1. Sumer, B.M., Fredsøe, J.: The mechanics of scour in the marine environement. World Scientific (2002)

2. Sánchez-Arcilla, A., Cáceres, I., van Rijn, L., Grüne, J.: Revisiting mobile bed tests for beach profile dynamics. Coastal Eng. 58(7), 583-593 (2011)

3. Sumer, B.M., Hatipoglu, F., Fredsöe, J.: Wave scour around a pile in sand, medium dense and dense silt. J. Waterway, Port, Coastal, Ocean Eng. 133(1), 14-27 (2007)

4. Astruc, D., Cazin, S., Cid, E., Eiff, O., Lacaze, L., Robin, P., Toublanc, F.: Cáceres: A stereoscopic method for rapid monitoring of the spatio-temporal evolution of the sand-bed elevation in the wash zone. Coastal Eng. 60(1), 11-20 (2012)

5. Baglio, S., Faraci, C., Foti, E., Musumeci, R.: Measurements of the 3-D scour process around a pile in an oscillating flow through a stereo vision approach. Measurement 30(2), 145-160 (2001)

6. Foti, E., Cáceres-Rabionet, I., Marini, A., Musumeci, R.E., Sánchez-Arcilla, A.: Experimental investigations of the bed evolution in wave flumes: Perrformances of 2D and 3D optical systems. Coastal Eng. 58(7), 606-622 (2011)

7. Sumer, B.M., Petersen, T.U., Locatelli, L., Fredsöe, J., Musumeci, R.E., Foti, E.: Backfilling of a scour hole around a pile in waves and current. J. Waterway, Port, Coastal, Ocean Eng. 139(1), 9-23 (2013)

8. Lowe, D.G.: Distinctive image features from scale-invariant key-points. International Journal of Computer Vision 60(2), 91-110 (2004)

9. Abdel-Aziz, Y.I., Karara, H.M.: Direct linear transformation into object space coordinates in close-range photogrammetry. In: Proc. Symposium in Close-Range Photogrammetry, Urbana, Illinois, pp. 1-18 (1971)

10. Faugeras, O.D., Toscani, G.: Camera calibration for 3D computer vision. In: Proc. International Workshop on Industrial Applications of Machine Vision and Machine Intelligence, Silken, Japan, pp. 240-247 (1987)

11. Heikkilä, J., Silvé, O.: A four-step camera calibration procedure with implicit image correction. In: IEEE Computer Society Conference on Computer Vision and Pattern Recognition, San Juan, Puerto Rico, pp. 1106-1112 (1997)

12. Tsai, R.Y.: A versatile camera calibration technique for hygh-accuracy $3 \mathrm{D}$ machine vision metrology using off-the shelf TV cameras and lenses. IEEE J. Robotics and Automation RA-3(4), 323-344 (1987) 
13. Allan, G.H., Serra, J.: Morphological operators on the unit circle. IEEE Transactions on Image Processing 10(12), 1842-1850 (2001)

14. Soille, P.: Morphological image analysis: Principle and Applications. Springer (1999)

15. Hartley, R., Zisserman, R.: Multiple view geometry in computer vision. Cambridge University Press (2003)

16. Newcombe, R.A., Izadi, S., Hilliges, O., Molyneaux, D., Kim, D., Davison, A.J., Kohli, P., Shotton, J., Hodges, S., Fitzgibbon, A.: KinectFusion: Real-Time Dense Surface Mapping and Tracking. In: IEEE International Symposium on Mixed and Augmented Reality (2011) 